\title{
Accessibilità post-pandemia: riflessioni sullo spazio pubblico
}

\author{
Giancarlo Gallitano \\ Dipartimento di Architettura, Università degli Studi di Palermo, Italia \\ giancarlo.gallitano@unipa.it
}

\section{Manfredi Leone}

Dipartimento di Architettura, Università degli Studi di Palermo, Italia manfredi.leone@unipa.it

\section{Francesca Lotta}

Dipartimento di Architettura, Università degli Studi di Palermo, Italia francesca.lotta@unipa.it

\begin{abstract}
The correlation between the psycho-physical well-being of citizens and the provision of green areas has been one of the cornerstones of urban planning since its inception, as its constant commitment to adapt cities and territories to emerging challenges. The spread of COVID-19 has changed the relationship between citizens and urban space. The public space has been limited and banned, confining citizens within their private space and the psycho-physical well-being of citizens depended only on the quality of the domestic space.

The pandemic represents a moment of reflection and research for the disciplines that deal with the quality of life of citizens.

The pandemic crisis may be an opportunity to rethink the role and importance of the provision of public space and urban green areas, their distribution and effective accessibility.

The paper analyzes current models of urban development as potential responses to the challenges of the post-pandemic city.
\end{abstract}

\section{Parole chiave}

Well-being, accessibility, public space.

\begin{abstract}
La correlazione tra il benessere psico-fisico dei cittadini e disponibilità di spazi pubblici a verde è stato uno dei cardini della pianificazione urbana sin dal suo esordio, così come il suo costante impegno per adattare città e territori alle sfide emergenti. La diffusione di COVID-19 ha cambiato il rapporto tra cittadini e spazio urbano. Lo spazio pubblico è stato limitato e interdetto, confinando i cittadini all'interno del loro spazio privato, facendo dipendere e il benessere psicofisico dei cittadini solo dalla qualità dello spazio domestico.

La pandemia rappresenta un momento di riflessione e ricerca per le discipline che si occupano della qualità della vita dei cittadini.

La crisi pandemica può essere un'opportunità per ripensare il ruolo e l'importanza della dotazione di spazi pubblici e aree verdi urbane, la loro distribuzione ed effettiva accessibilità. Il contributo analizza attuali modelli di sviluppo urbano come potenziali risposte alle sfide della città post-pandemica.
\end{abstract}

\section{Parole chiave}

Benessere, accessibilità, spazio pubblico 


\section{COVID-19: prospettive per la ricerca}

La pandemia in atto rappresenta un momento di riflessione e ricerca per le discipline che si occupano della qualità della vita dei cittadini, non ultime le scienze sociali e quelle afferenti alla progettazione e pianificazione urbana e paesaggistica. C'è grande incertezza su quale sarà l'impatto finale del coVID-19 sulla progettazione, l'uso e la percezione dello spazio pubblico; su come cambierà il nostro rapporto con esso e per quanto tempo dovremo convivere con tali cambiamenti e, se questi diventeranno permanenti, anche in termini di nuovi comportamenti sociali. Sono molte le domande in sospeso che diversi autori (Florida, 2020; Markusen, 2020; Roberts, 2020) si stanno ponendo per iniziare a delineare futuri scenari, consapevoli che si potranno verificare nuove epidemie e nuovi periodi di distanziamento sociale (Kissleret al., 2020).

Rispetto al campo disciplinare della progettazione e pianificazione urbana e paesaggistica, concentrandosi sull'esperienza italiana, emergono almeno tre prospettive di ricerca.

La prima prospettiva è quella ambientale. A una prima lettura della diffusione del COVID-19 sul territorio nazionale è emersa una corrispondenza tra la mappa della diffusione del contagio e quella della presenza di aree industriali e, conseguentemente, dell'inquinamento atmosferico.
Al tempo stesso, la ridotta presenza dell'uomo, per le misure restrittive adottate, ha permesso di compiere osservazioni sulla velocità dei sistemi naturali di riappropriarsi dei suoli urbanizzati: animali selvatici per le città deserte e livelli di disinquinamento di aria e acqua. In una ristretta finestra temporale, ha avuto luogo una sorta di esperimento globale involontario sulle dinamiche ambientali in assenza di abitanti. Secondo questa prospettiva di ricerca, l'attuale pandemia rappresenta una nuova opportunità per integrare prospettive di benessere psico-fisico degli abitanti nella progettazione urbana. II miglioramento delle condizioni sanitarie delle città ha motivato pianificatori, architetti e ingegneri a riprogettare le città già alla fine deI XIX secolo (Sennett, 2018). Pandemia e misure restrittive determinano una seconda prospettiva di ricerca sul nuovo rapporto tra abitanti e spazio pubblico in termini di rimodulazione dello spazio fisico e delle sue funzioni, secondo principi di temporaneità e flessibilità. Le norme per il contenimento della pandemia hanno prodotto la rarefazione della sfera relazionale pubblica e un nuovo livello di domanda spazio-temporale per la fruizione dello spazio pubblico. Questo permette di accedere a un sistema eterogeneo di beni e servizi, assolvendo a funzioni sociali, ricreative, culturali e commerciali. Affinché lo spazio pubblico possa continuare ad assolvere a tali funzioni senza rinun- 


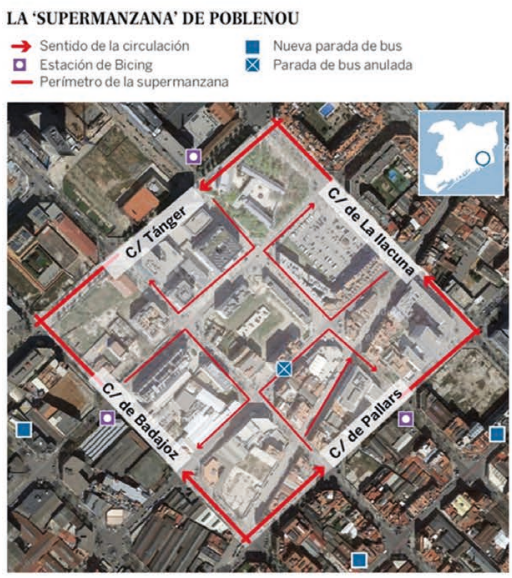

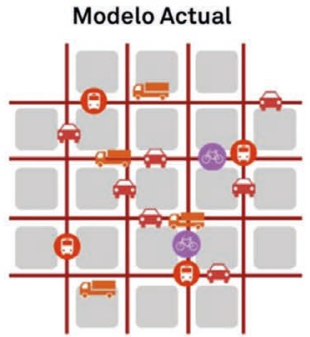

Modelo Supermanzanas

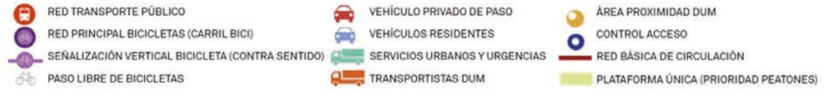

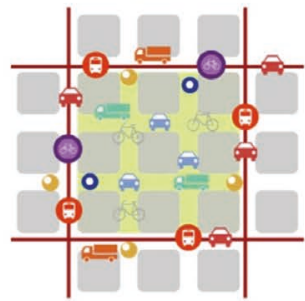

PLATAFORMAÚNICA (PRIORIDAD PEATONES

Fig. 1 - II modello Supermanzanas e un caso applicativo nel quartiere Poblenou a Barcellona (fonte: Ajuntamento de Barcelona)

ciare alla sua complessità, è necessario che a una sua dilatazione spaziale corrisponda una rimodulazione in termini di giusto equilibrio tra temporalità e contemporaneità degli usi. II rischio è ridurre la complessità dello spazio pubblico a rigidi schemi funzionali e diagrammi orari. Blocchi e limitazioni di servizi e attività non essenziali hanno determinato una improvvisa riduzione del traffico veicolare. Oltre il periodo di lockdown, restrizioni secondarie continuano a mantenere un livello dei flussi veicolari ridotti e limitano fortemente la portata dei servizi di trasporto pubblico di massa.

Pertanto, una terza prospettiva di ricerca indaga i nuovi scenari della mobilità urbana, in particolare il rapporto tra mobilità individuale e collettiva, influenzata dalle misure restrittive. Se da un lato la riduzione dei flussi veicolari ha concesso nuovi spazi a ciclisti e pedoni, limitando le interferenze tra le diverse forme di mobilità, le limitazioni ai trasporti pubblici hanno generato un nuovo livello di domanda di mobilità dolce. La domanda è stata ulteriormente alimentata dalla decisione del governo nazionale di offrire incentivi per l'acquisto di mezzi di 244 trasporto individuali sostenibili.
Ciò evidenzia come il COVID-19 si inserisca all'interno del più ampio processo di transizione globale verso la sostenibilità ambientale. Questo tipo di transizione è già in atto in diverse grandi città. Ne è un esempio Barcellona dove, dopo le prime Supermanzanas (Rueda, 2002, 2019) previste dal Piano urbano della mobilità, il divieto al traffico di auto si estenderà ad altre zone. Al posto di 21 incroci si avranno 21 nuove aree pedonali. Per l'attuazione del piano sarà avviata una competizione pubblica per la progettazione, con pochi punti fermi: l' $80 \%$ della superficie stradale dovrà essere ombreggiata e il 20\% di superficie dovrà essere permeabile e in grado di raccogliere l'acqua piovana, aumentando la resilienza in caso di alluvioni. II presente contributo, nel ricomporre le tre prospettive di ricerca, si concentra sulla dotazione di aree verdi e sulla loro accessibilità in termini di benessere psico-fisico e giustizia spaziale.

\section{Pandemia, accessibilità e fruizione dello spazio urbano}

La città della pandemia è una città basata sul distanziamento sociale. Le misure di limitazione della diffusione del COVID-19 hanno ridotto o vietato 

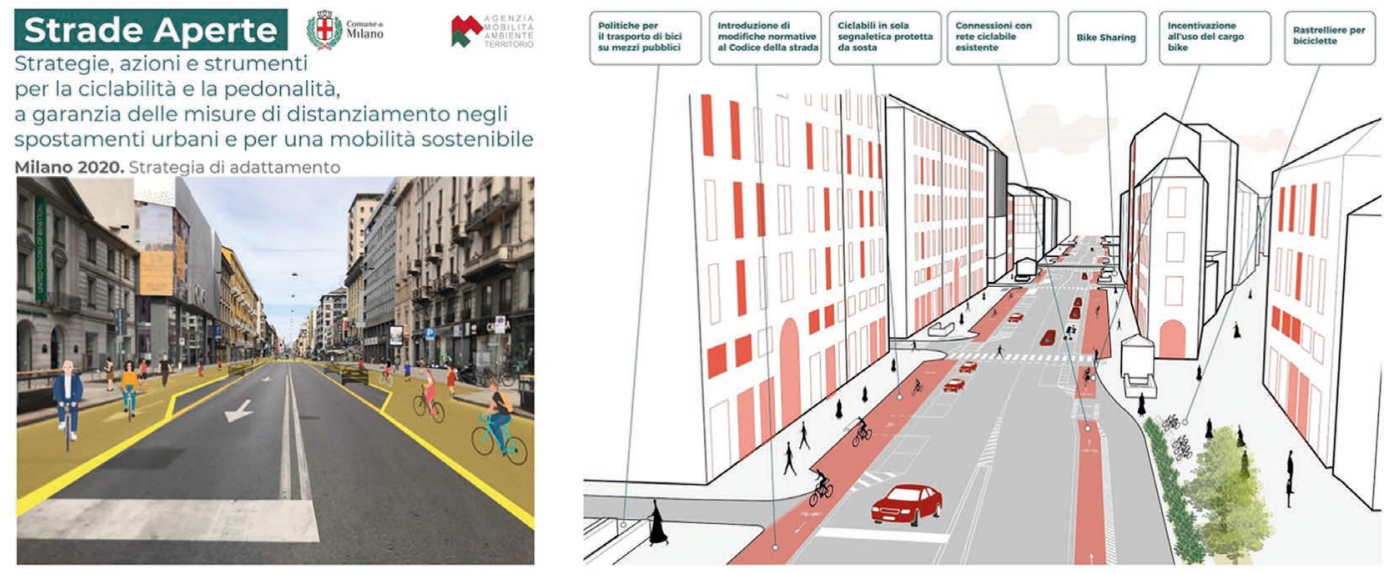

Fig. 2 - Documento programmatico Strade Aperte: focus sull'intervento su Corso Buenos Aires (fonte: Comune di Milano).

la fruizione degli spazi pubblici, generando una crisi dello spazio pubblico e della vita associata, dettata dallo stato di emergenza. La densa e costretta domesticazione dello spazio e del tempo (Ehn e Löfgren, 2010) ha reso necessaria una riorganizzazione tempestiva della nostra sfera sociale e del nostro rapporto con lo spazio pubblico (Florida, 2020; Null e Smith, 2020; Roberts, 2020; van der Berg, 2020). Già nel 2015, a conclusione del Summit sullo Sviluppo Sostenibile, i membri delle Nazioni Unite hanno sottoscritto il programma di azione conosciuto come Agenda 2030. Tra i 17 Sustainable Development Goals, I'undicesimo recita: "Rendere le città e gli insediamenti umani inclusivi, sicuri, duraturi e sostenibili" e propone di "fornire accesso universale a spazi verdi e pubblici sicuri, inclusivi e accessibili, in particolare per donne, bambini, anziani e disabili" ? L'organizzazione per gli spazi pubblici e verdi nell'ambito degli insediamenti urbani è questione antica, molto più di quanto il campo disciplinare dell'urbanistica affermi quando fa coincidere la nascita della questione con le origini della città industriale.

Spesso il tema della presenza del verde in città ha mosso politiche e definito scelte urbanistiche e pro- gettuali di cui riconosciamo ancora oggi lo spessore culturale, come il concetto di greenbelt che rispondeva a differenti problematiche: il sovraffollamento delle città vittoriane analizzato da Howard; le esigenze ricreative che interessarono Unwin e la crescita urbana sregolata affrontata da Abercrombie.

Lo spazio verde e pubblico è sempre stato considerato un fondamento del nostro benessere. Lo sapeva bene Olmsted (1865) che nella relazione progettuale per il Central Park di New York, sottolineava le positive implicazioni psicologiche. Tali implicazioni saranno rafforzate da studiosi come Norberg-Schulz che, nell'affermare lo spazio urbano quale elemento esistenziale dell'abitare (1979), disquisisce sul concetto di orientamento, identificazione e sicurezza percepita. Egli, facendo leva sugli studi scientifici della Gestalt e della psicologia della percezione di Piaget, rimanderà più volte all'importanza del riconoscimento del luogo (1969, p. 21). Inoltre, nell'ambito della psicologia ambientale, ai benefici fisici e psicologici individuali, come la riduzione dello stress (Ulrich, 1983, Ulrich et al., 1991; Grahn e Stigsdotter, 2003; van derBerget al., 2007), si aggiungono quelli di comunità, come l'aumento 


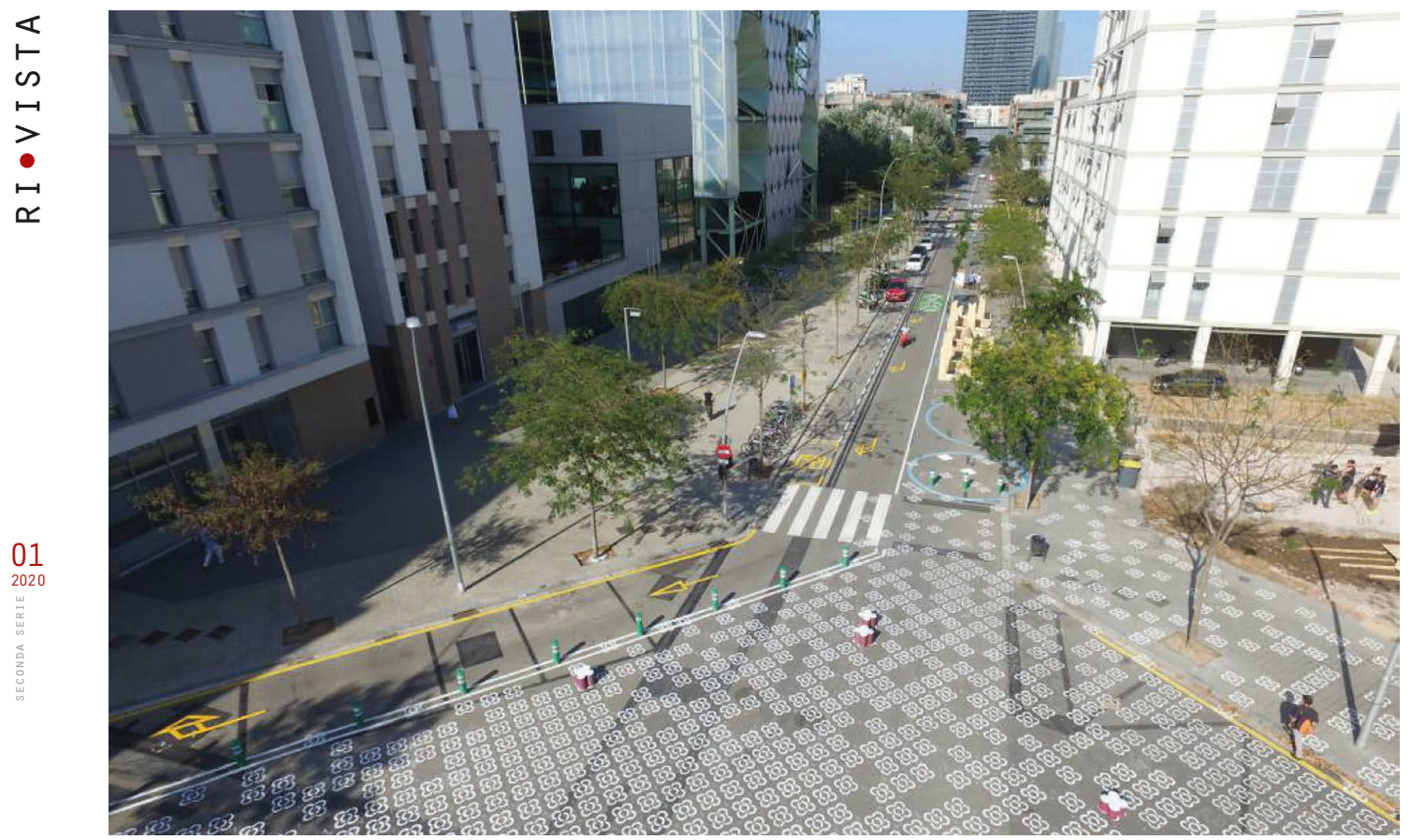

Fig. 3 - Uno degli incroci trasformato in piazza in occasione delle sperimentazioni condotte nel settembre del 2016 (fonte: El Pais, https://elpais.com/elpais/2019/10/07/icon_design/1570456123_584326.html).

della coesione sociale (Coleyet al., 1997; Kuo e Sullivan, 2001).

La sfida è dunque capire come l'agire progettuale possa rendere ancora praticabile lo spazio pubblico, equilibrando ed eventualmente integrando fra loro funzioni esterne e interne allo spazio privato. Da questo punto di vista, le sfide urbane da affrontare sono principalmente due: strutturare un sistema di mobilità sostenibile alternativo al trasporto pubblico di massa; garantire l'accessibilità e la fruizione degli spazi aperti a tutti. Come espresso precedentemente, non sono nuove sfide ma nuovo è il livello e il tipo di domanda che le restrizioni hanno determinato. La crisi pandemica può essere letta come un'opportunità per aumentare lo spazio destinato a pedoni e ciclisti, lavorando in prospettiva dell'obiettivo di abitare città più verdi (Nieuwenhuijsen, 2020; Roberts, 2020). Sin dai primi giorni della crisi si è discusso sul- disfare le norme sul distanziamento sociale. Mentre New York City stava valutando di fare una simile mossa (Bliss, 2020), Milano ${ }^{3}$ è stata una delle prime città ad avviare cambiamenti permanenti, ampliando lo spazio per i pedoni e pianificando $35 \mathrm{~km}$ di nuove piste ciclabili, a discapito delle corsie per i veicoli. Anche Boston, Londra, Portland e Vancouver hanno avviato iniziative analoghe (Hawkins, 2020; Topham, 2020).

Per il caso di Barcellona, le sperimentazioni nel quartiere Poblenou si sono attuate attraverso laboratori creativi aperti alla cittadinanza, in azioni di tactical urbanism per la conversione dello spazio carrabile in spazio pubblico pedonale. Si è fatto ricorso all'uso di un elemento riconoscibile di grafica pavimentale che disegna uno spazio immaginario e simbolico, rafforzando il senso di appartenenza e riappropriazione dello spazio urbano. Attraverso il riuso di materiali di scarto, sono state individuate aree con 
Gathering area provision $\left(\mathrm{m}^{2}\right)$ Dotazione di aree recreative $\left(\mathrm{m}^{2}\right.$

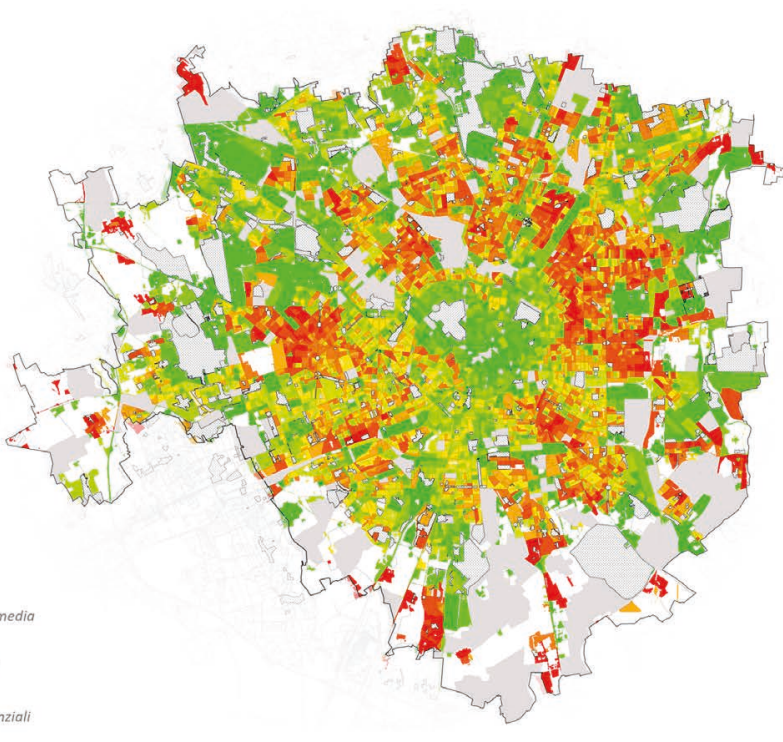

653,000

Inhabitants of scarcely served areas Abitanti in aree scarsamente servite

\section{3,000}

Inhabitants of medium served areas

Abitanti in aree

mediamente servite

294,000

Inhabitants of highly

served areas

Abitanti in aree

altamente servite

Fig. 5 - Mappa della dotazione di aree ricreative della città di Milano (fonte sistemica 2020, <https://research.systematica.net/ research/access-to-green-areas-and-public-realm-the-case-of-milan/>).

Tali considerazioni sono vere solo se gli spazi verdi sono realmente accessibili e fruibili.

In relazione all'accessibilità e alla fruizione delle aree verdi, dopo la fase di totale chiusura di parchi e giardini e di limitazione delle attività sportive all'aperto, le amministrazioni cittadine hanno consentito ingressi contingentati (spesso su prenotazione) e brevi passeggiate all'aperto in prossimità delle abitazioni. Ciò ha determinato l'insorgere di un nuovo tipo di domanda prestazionale in aggiunta a quella di reale qualità degli spazi che ne garantisca un'attrattività e un livello di fruizione adeguato.

Una delle configurazioni fisiche che meglio risponde alle sopracitate necessità è la strutturazione in rete. II concetto di rete affida al lemma tecnico 'infrastruttura' tale articolazione. Tale declinazione terminologica si è sviluppata in una prospettiva ecologico-ambientale nel contesto americano di fine ‘800, continuando a essere riconfermata dalla U.S. Environmental Protection Agency (EPA) e da ricerca-
Così come l'infrastruttura dei trasporti è costituita da una rete di strade, ferrovie e aeroporti, allo stesso modo l'infrastruttura verde ha tra le sue componenti parchi, fiumi e alberature stradali. L'infrastruttura verde, estendendo l'attenzione finanche ad aspetti paesaggistici (Peraboni, 2010), come sottolineato recentemente dalla Risoluzione del Parlamento Europeo del 17 settembre $2020^{5}$, può essere la risposta a più sfide: dall'impatto dei cambiamenti climatici sulla salute dei residenti, alle tematiche ambientali e ancora allo stesso fronteggiare la pandemia COVID-19. Tale dispositivo progettuale si prefigge di andare oltre la categoria generica e puntuale del verde urbano, dislocando in modo capillare i suoi spazi, spesso rafforzati anche da elementi grigi come i servizi pubblici (Angrilli, 2002) con il fine di aumentare la presenza di aree verdi accessibili.

In questa prospettiva, gli spazi di pertinenza degli edifici pubblici potrebbero rappresentare una importante risorsa anche in un periodo pandemico. Infatti, solitamente queste aree hanno punti di acces- 


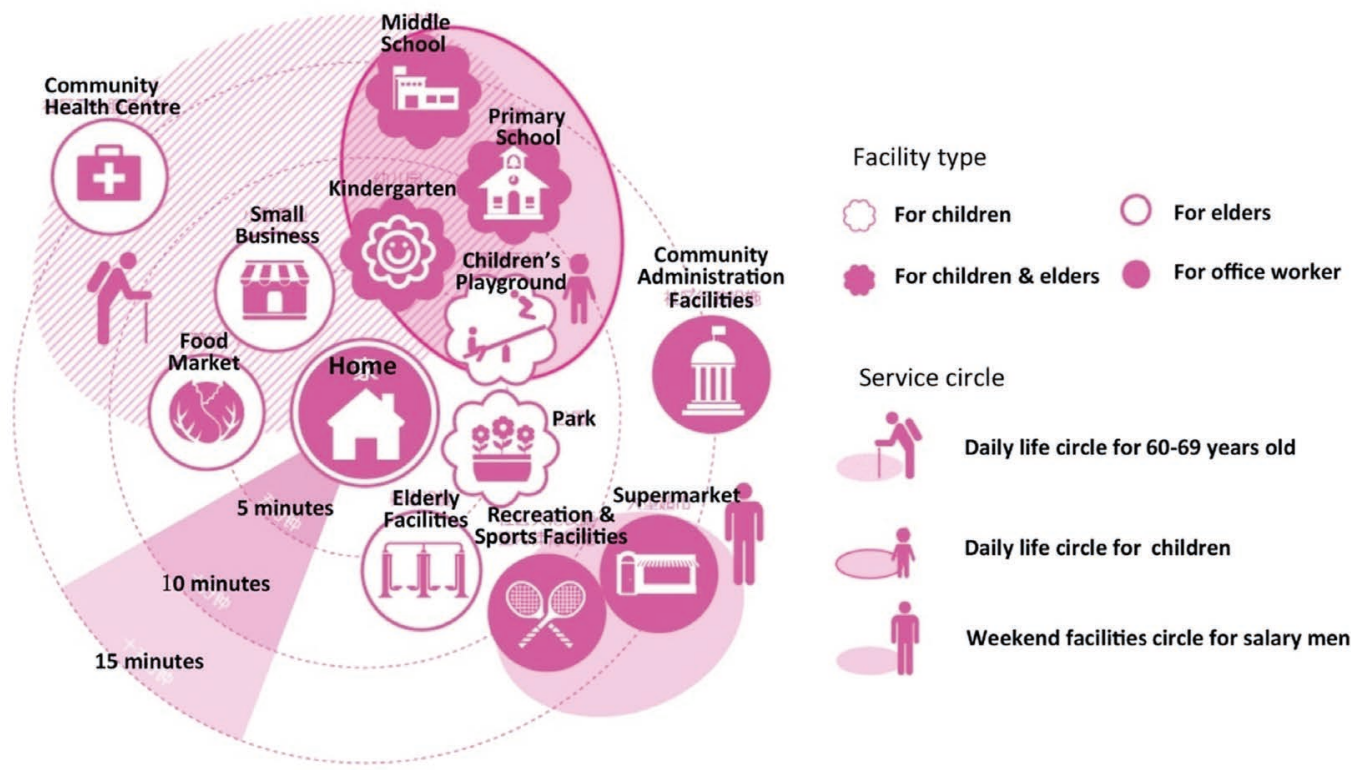

Fig. 6 - II paradigm '15 minutes compact city neighbourhood' (fonte: Shanghai Urban Planning and Land Resources Administration Bureau, 2016, citato in Hou e Yungang, 2017, p. 8).

so limitati, controllabili, che possono garantire un afflusso regolato di fruitori. La loro distribuzione sul territorio, si pensi alle scuole, garantisce una implementazione abbastanza omogenea della dotazione di aree verdi, soprattutto a scala di quartiere. In più, queste aree godono spesso di una buona accessibilità per vicinanza alla rete stradale primaria che in accordo con gli strumenti di programmazione di settore, potrebbe essere messa in rete con piste ciclabili già esistenti.

\section{Comprendere il nuovo rapporto domanda/offerta} In accordo con il documento dell'Agenzia delle $\mathrm{Na}$ zioni Unite UN-Habitat's key messages on COVID-19 and public space contenente indicazioni di carattere generale per un'effettiva risposta alle criticità derivanti dal COVID-19 in ambito urbano, tre sono i principali studi necessari per quantificare la nuova domanda di fruizione di spazio pubblico e predisporre strategie di offerta di breve, medio e lungo termine. Nello specifico bisogna:
- condurre analisi spaziali a livello urbano, finalizzate a mappare le risorse ed evidenziare i punti di debolezza;

- comprendere tutte le funzioni a cui rispondono gli spazi aperti in città;

- ripensare alla dotazione di spazio pubblico in termini di temporaneità, flessibilità e multifunzionalità.

Rispetto al primo punto, appare interessante l'esperienza di ricerca condotta da Sistemica (2020) per il Comune di Milano. Lo studio segue un approccio analitico volto ad affrontare la transizione della città verso un'offerta di spazio pubblico più ampia e omogenea. Consapevoli che l'attuale pandemia sta cambiando i comportamenti sociali consolidati, soprattutto in ambito urbano dove è avvertita la necessità di spazi di aggregazione all'aperto, è necessario operare una loro rimodulazione, migliorando l'accessibilità e la prossimità. Una delle mappe elaborate grazie a un'analisi isocronica definisce proprio un indice di accessibilità. 

ziale, è uno degli elementi centrali dell'idea di città compatta ed è stato ampiamente identificato come meccanismo utile per raggiungere obiettivi di sostenibilità urbana tra cui la vitalità, l'uso efficiente dei servizi urbani e la coesione sociale (Foord, 2010). Tuttavia, nonostante sia considerato più sostenibile dal punto di vista ambientale, un ambiente urbano compatto è spesso associato a una minore vivibilità. Questo "compact city paradox" (Neuman, 2005) si basa sulla percezione comune che la città compatta implichi diversi effetti negativi ed è stato supportato da vari studi teorici (Wirth, 1938; Fischer, 1973) ed empirici (Rodgers, 1981; Bramley et al., 2009). In questo senso, l'esperienza della pandemia potrebbe dissipare dubbi e promuovere lo sviluppo di quartieri 'autonomi' legati alla dimensione dell'abitare.

Nella dimensione dei singoli spazi e attrezzature, la città post-pandemica li progetta e realizza in termini di flessibilità, multifunzionalità e su un uso temporaneo (in relazione alle diverse funzioni da accogliere), comprese le strade. Queste, a ridotto o nullo traffico veicolare, potrebbero essere adattate in spazi pubblici confortevoli con semplici interventi di coloritura del manto stradale e l'inserimento di nuovi arredi, coinvolgendo gli abitanti in processi di rigenerazione urbana a scala di quartiere.
Ne è un esempio il progetto Piazze aperte del Comune di Milano, antecedente alla crisi pandemica. Non è solo la mobilità ciclo-pedonale e la dimensione ricreativa dello spazio pubblico a dover attrarre l'attenzione di pianificatori e progettisti. Marciapiedi e piazze, soprattutto nel sud del mondo, tendono a essere affollati, spesso occupati da venditori ambulanti e da altre attività informali. Lo spazio pubblico, infatti, si configura anche come luogo necessario al sostentamento delle fasce più deboli della popolazione urbana. Welfare e città è stato un binomio fondamentale nel progetto della città del XX secolo e deve tornare a esserlo perché l'attuale situazione sociale mette ancora più in evidenza la fragilità economica di un gran numero di cittadini, accentuata dalla crisi finanziaria del 2008 e dal fatto che le politiche di sostegno economico non possono bastare a ritrovare un senso di benessere. Se dunque un racconto importante per la città del XX secolo è stato quello di una ricerca paziente delle dimensioni fisiche e concrete del benessere individuale e collettivo (Secchi, 2005), oggi è importante tornare a riflettere 'concretamente' sulla costruzione fisica, quindi urbana, del benessere collettivo e riprogettare lo spazio urbano nella sua totalità.

Anche le strade possono essere ripensate per soddisfare altre esigenze emergenti oltre al distanziamento sociale. Ad esempio, la crisi pandemica ha interessato anche i settori dello sharing e del delivery. Se è vero che lo shopping online e i servizi di delivery hanno conosciuto un aumento nella domanda, tale incremento ha però creato ritardi nei tempi di consegna facendo preferire metodi di acquisto tradizionali, soprattutto per i beni di prima necessità. Al contempo, i mercati tradizionali, non potendo garantire opportune condizioni di sicurezza sono stati chiusi, mentre gli accessi ai centri della grande distribuzione erano contingentati. Pertanto, strade e piazze potrebbero anche essere adattate per ospitare aree di mercato, decongestionando quelle esistenti. 


\section{Visioni per la città post-pandemica}

Dimensioni, portata e velocità della pandemia testimoniano che stiamo vivendo una profonda trasformazione: un cambiamento tettonico, in cui il terreno si muove sotto di noi, cambiando i principi e le regole fondamentali che hanno governato le pratiche del quotidiano. Si tratta di cambiamenti di paradigma (Kuhn, 1962). Questi momenti di rottura sono opportunità per intraprendere progetti radicalmente nuovi e audaci; creano opportunità per svolgere attività precedentemente ritenute impossibili ma ora necessarie. Non è chiaro se gli impatti del COVID-19 sullo spazio pubblico saranno profondi quanto lo sono in altri aspetti della nostra vita (Corbera et al., 2020). La vera domanda è per quanto tempo questi impatti saranno avvertiti e quale sarà la loro portata trasformativa. Potrebbero essere necessari anni prima di poter accertare come la pandemia abbia cambiato la pianificazione e la progettazione dello spazio pubblico.

La città post-pandemica attua contemporaneamente strategie di breve periodo, secondo interventi tipici del tactical urbanism (Lydon e Garcia, 2015) e di medio-lungo periodo abbracciando la sfide della sostenibilità ambientale, questione che, negli ultimi vent'anni, ha sempre più preso piede nella prassi urbanistica, anche focalizzandosi sull'aumento della 'resilienza socio-spaziale', intesa come “de-centered, de-commodified and de-carbonised alternative" (Brown, 2011, p. 14), garantendo che le diverse comunità abbiano gli strumenti per affrontare l'emergenza. In questo senso il modello del '15 minutes compact city neighbourhood' potrebbe rappresentare un obiettivo di lungo periodo.

II nuovo livello di domanda di spazi aperti e di mobilità ciclo-pedonale, però, non può essere risolto da un punto di vista puramente quantitativo. La questione della prossimità e dell'effettiva capacità di accogliere funzioni e utenti, vanno associate alla reale qualità dei luoghi che ne garantisca un'attrattività e un livello di fruizione adeguati.
L'incremento degli spazi aperti disponibili e una loro migliore distribuzione costituirebbe certamente una opportunità a fronte delle limitazioni, contrastando le condizioni di isolamento durante i lockdown.

La città post-pandemica progetta e realizza spazi e attrezzature flessibili, basati su un modello multifunzionale e su un uso temporaneo, legato alla temporalità delle funzioni che possono accogliere, comprese le strade.

Al tempo stesso, spazi e attrezzature dovranno essere progettati per rispondere agli obblighi di distanziamento e regolazione dei flussi; dovranno rispondere a nuovi parametri di qualità nell'uso dei materiali non solo sostenibili, ma anche idonei alla frequente sanificazione; dovranno prevedere sistemi di gestione e manutenzione garanti della salute dei cittadini e della continuità dei servizi.

Lo spazio urbano, così come immaginato da Tom Turner nel suo libro City as landscape (1996) sarebbe contraddistinto e caratterizzato da numerosi colori che rimanderebbero a una prevalenza funzionale, mutevole secondo della necessità. Le nostre città, seppur 'depotenziate', muterebbero con ironico compiacimento in spazi arlecchino, esito del sovrapporsi e del coesistere di funzioni e attività differenti.

\section{Considerazioni conclusive}

La riflessione proposta mette in luce alcune prospettive di cambiamento che già sono in atto ma che la diffusione del COVID-19 ha accelerato.

Le restrizioni adottate per il contenimento della pandemia hanno inciso sulle routines individuali e collettive - intese come elementi di stabilità e stabilizzazione dei modelli sociali (Ehn e Löfgren, 2010) ma anche pratiche agenti, volte a ridefinire lo spazio (De Certeau, 2012) -, hanno incentivato alcune dinamiche già in atto come la trasformazione temporanea di strade in piazze, attraverso azioni di tactical urbanism o come l'aumento della domanda di mobilità sostenibile individuale ha spin- 
to a una ridefinizione del rapporto tra spazio destinato alle auto e quello destinato a ciclisti e pedoni. Lo sforzo globale, seppur differenziato, ha condotto a sperimentazioni seguendo decisioni 'tattiche' sull'uso/rimodulazione dello spazio pubblico. Si è trattato di tentativi di salvataggio della dimensione pubblica delle città attraverso la riattivazione di sinergie tra individui di una collettività locale che condivide il medesimo territorio dell'abitare. È stato anche un tentativo di preservare il benessere psico-fisico degli abitanti, dato che il legame con lo spazio rientra tra i bisogni fondamentali degli esseri umani. Lo sforzo per ri-definire l'uso, adattare e ri-progettare lo spazio e le attrezzature non può però essere soltanto legato all'emergenza. Se è vero che in una fase iniziale è necessario individuare luoghi idonei per organizzare la distribuzione di alimenti, insediare centri di emergenza sanitaria, svolgere attività fisica all'aperto o a ospitare mercati rionali, è anche vero che nella locuzione 'post-pandemica' è implicito un concetto di radicale cambiamento e il COVID-19 rappresenta uno spartiacque nel modo di percepire e vivere lo spazio pubblico.

Pianificazione e progetto degli spazi aperti dovrebbero non solo promuovere la capillarizzazione di questi spazi, ma migliorarne la qualità e soprattutto l'accesso. È in questo contesto che, ancora una vol- banistico chiaro, quello di infrastruttura verde urbana. Incrementando le consuete tipologie dello spazio urbano riscontrabili nella lettura di diversi manuali scientifici, l'infrastruttura verde urbana sarebbe quell'elemento che consentirebbe lo sviluppo di relazioni ecologiche della città con il proprio contesto ambientale e, allo stesso tempo, il soddisfacimento delle istanze sociali e del welfare, fondamentali per il conseguimento di un'elevata qualità urbana anche in un periodo pandemico.

II tentativo di adattare le città, può rappresentare un'occasione per ripensare il rapporto tra uomo e natura e porre nuova attenzione ai temi della sostenibilità e del cambiamento climatico. La pandemia, pertanto, rappresenta uno sfondo operativo inaspettato che obbliga il ripensamento tempestivo di tattiche e strategia per la pianificazione dello spazio pubblico e del paesaggio, secondo nuove possibili dinamiche future, attraverso una necessaria rivisitazione del rapporto abitanti/luoghi e - forse soprattutto - attraverso una rinvigorita dimensione di temporaneità, in termini di precarietà/flessibilità, come mai prima d'ora si è verificato nella storia urbana in tempi di 'pace'. 


\section{Note}

${ }^{1}$ Benché questo contributo possa essere considerato il risultato delle comuni riflessioni degli autori, ai fini dell'attribuzione i paragrafi $1^{\circ} \mathrm{e} 4^{\circ}$ si devono a Manfredi Leone, il $2^{\circ}$ a Francesca Lotta e il $3^{\circ}$ a Giancarlo Gallitano. Le conclusioni sono state scritte congiuntamente dai tre autori.

2Documento consultabile sul sito dell'Agenzia per la Coesione territoriale al seguente link: <https://www. agenziacoesione.gov.it/comunicazione/agenda-2030-per-lo-sviluppo-sostenibile/> (07/20).

${ }^{3}$ Milano ha elaborato, coinvolgendo i cittadini, una strategia per la cosiddetta 'Fase 2', Milano 2020, strategia di adattamento, all'interno del quale è si inserisce il progetto Strade Aperte, ed ha redatto il Piano di azione per la mobilità urbana post COVID, denominato 'Rete di mobilità d'emergenza'. ${ }^{4}$ Lo studio è consultabile al seguente link: <https://www. Iondon.gov.uk/what-we-do/environment/parks-green-spaces-and-biodiversity/green-infrastructure/natural-capital-account-london? source=vanityurl> (03/21).

${ }^{5}$ Risoluzione del Parlamento europeo del 17 settembre 2020 sull'anno europeo delle città più verdi 2022 (2019/2805(RSP)), <https://www.europarl.europa.eu/doceo/document/TA-9-2020-0241_IT.html> (03-21).

${ }^{6}$ L'obiettivo è fornire servizi pubblici di base e servizi commerciali entro 15 minuti a piedi per ciascuna comunità. In questo modo, verrebbe promosso anche uno stile di vita più sano. II calcolo per la definizione delle distanze considerare le diverse caratteristiche comportamentali dei diversi gruppi (ad es. l'intera popolazione, bambini, adulti e anziani) e le caratteristiche delle attrezzature (scala e categoria), cfr. Wenget al. 2019.

${ }^{7}$ Piazze aperte rientra nel Piano periferie; è un progetto del Comune di Milano che utilizza l'approccio del tactical urbanism, coinvolgendo gli abitanti nei processi di rigenerazione urbana in interventi spaziali e politiche a breve termine, a basso costo e scalabili per riportare lo spazio pubblico al centro della vita del quartiere. II progetto ha un carattere sperimentale e temporaneo. <https://www.comune.milano.it/aree-tematiche/quartieri/piano-quartieri/piazze-aperte $>(07 / 20)$

\section{Bibliografia}

Angrilli M. 2002, Reti verdi urbane, Fratelli Palombi Editore, Roma.

Benedict M., McMahon E.T. 2002, Green Infrastructure: Smart Conservation for the 21st Century, «Renewable Resources Journal», vol. 20, n. 3, pp. 12-17, <http://www. greeninfrastructure.net/sites/greeninfrastructure.net/ files/GI_RR.pdf> (03/20).

Bliss L. 2020, Mapping How Cities Are Reclaiming Street Space, «CityLab», <https://www.citylab.com/transportation/2020/04/coronavirus-city-street-public-transitbike-lanes-covid-19/609190/> (07/20).

Bramley G. et al. 2009, Social sustainability and urban form: Evidence from five British cities, «Environment and Planning A», n. 41, pp. 2125-2142.

Brown K. 2011, Rethinking Progress in a Warming World: Interrogating Climate Resilient Development. Conference "Rethinking development in an age of scarcity and uncertainty: New values, voices and alliances for increased resilience". University of York, 19-22 September 2011.

Coley R.L., Sullivan W.C., Kuo F.E. 1997, Where does community grow? The social context created by nature in urban public housing, "Environment and Behavior», n. 29, pp. 468-494. 
01
Costanza R., d'Arge R., deGroot R., Farber S., Grasso M., Hannon B., Limburg K., Naeem S., Oneill R.V., Paruelo J., Raskin R.G., Sutton P., van den Belt M. 1997, The value of the world's ecosystem services and natural capital, «Nature», n. 387, pp. 253-260.

Ehn B., Löfgren 0. 2010, The secret world of doing nothing, California University Press, Berkeley.

Fischer CS. 1973, Urban malaise, «Social Forces»,n. 52, pp. 221-235.

Florida R.2020, We'll Need To Reopen Our Cities. But Not Without Making Changes First, «CityLab», <https://www.citylab.com/equity/2020/03/coronavirus-cities-adapt-future-plan-economy-infrastructure/608908/>(07/20).

Foord J. 2010, Mixed-use trade-offs: how to live and work in a compact city neighbourhood, «Built Environment», vol. 1, n. 36, pp. 47-62.

Grahn P., Stigsdotter U.A. 2003, Landscape planning and stress, «Urban Forestry and Urban Greening», vol. 1, n. 2, pp. 1-18.

Hawkins A.J. 2020, There's no better time for cities to take space away from cars, «Verge», <https://www.theverge. com/2020/3/23/21191325/cities-car-free-coronavirus-protected-bike-lanes-air-quality-social-distancing> (07/20).

Hou L., Yungang L. 2017, Life Circle Construction in China under the Idea of Collaborative Governance: A Comparative Study of Beijing, Shanghai and Guangzhou, «Geographical review of Japan series B», n. 90, pp. 2-16.

Kissler S.M., Tedijanto C., Goldstein E., Grad Y.H., Lipsitch M. 2020, Projecting the transmission dynamics of SARSCoV-2 through the postpandemic period, "Science», $\mathrm{n}$. 368, pp. 860-868.
Kuo F., Sullivan W. 2001, Environment and Crime in the Inner City: Does Vegetation Reduce Crime?, «Environment and Behavior», n. 33, pp. 343-367.

Lydon M., Garcia A. 2015, Tactical Urbanism, Island Press, Washington, DC.

Markusen A. 2020, Will COVID-19 drive us farther apart, or bring us together? Will we all move away from each other? Not likely, «Minnesota Reformer», <https://minnesotareformer.com/2020/04/07/will-covid-19-virusdrive-us-farther-apart-or-bring-us-together/> (07/20).

MEA-Millennium Ecosystem Assessment (2005), Ecosystems and Human Well-being: Synthesis, Island Press, Washington D.C..

Neuman M. 2005, The compact city fallacy, "Journal of Planning Education and Research», n. 25, pp. 11-26.

Nieuwenhuijsen M.J. 2020, Urban and transport planning pathways to carbon neutral, liveable and healthy cities; A review of the current evidence, «Environment International», vol. 140, 105661.

Norberg-Schulz C. 1969, I/ concetto di luogo, «Controspazio», n.1, p. 21.

Norberg-Schulz C. 1979, Genius Loci. Paesaggio, Ambiente, Architettura, Electa, Milano.

Null S., Smith H. 2020, COVID-19 Could Affect Cities for Years. Here Are 4 Ways They're Coping Now, "TheCityFix», World Resource Institute (WRI), Washington.

Olmsted Law F. 1865, Yosemite and the Mariposa Grove: A Preliminary Report, http://www.yosemite.ca.us/history/ olmsted/report.html (07/20).

Peraboni C. 2010, Reti ecologiche e infrastrutture verdi, Maggioli Ed., Santarcangelo di Romagna.

Roberts D.2020, How to make a city livable during lockdown, "Vox»,<https://www.vox.com/cities-and-ur- 
banism/2020/4/13/21218759/coronavirus-cities-lockdown-covid-19-brent-toderian> (08/20).

Rodgers WL. 1981, Density, crowding, and satisfaction with the residential environment, "Social Indicators Research», n. 10, pp. 75-102.

Rueda S. 2002, Barcelona, ciudad mediterránea, compacta y compleja, in Plan de movilidad, accesibilidad y espacio público en el Distrito de Gracia. Barcelona, Barcelona, Ajuntament de Barcelona.

Rueda S. 2019, Superblocks for the Design of New Cities and Renovation of Existing Ones: Barcelona's Case, in Integrating Human Health into Urban and Transport Planning, a cura di Nieuwenhuijsen M., Khreis H., Springer, Cham, pp.135-153.

Secchi B. 2005, La città del ventesimo secolo, Editori Laterza, Roma Bari.

Sennett R. 2018, Building and Dwelling: Ethics for the City, Penguin Random House, London, UK.

Topham G. 2020, London pedestrians and cyclists may get more space on roads, «The Guardian», <https:/ /www. theguardian.com/uk-news/2020/apr/14/london-pedestrians-and-cyclist-may-get-more-space-on-roadsduring-coronavirus-lockdown> (07/20).

Turner T. 1996, City as Landscape: A Post-postmodern View of Design and Planning, Taylor \& Francis, London.

Ulrich R. S. 1981, Natural versus urban scenes: Some psychophysiological effects. «Environment and Behavior», vol. 5, n.13, pp. 523-556.

Ulrich R.S. 1983, Aesthetic and affective response to natural environment, in Behavior and the natural environment, a cura di I. Altman, J.F. Wohlwill, Plenum Press, New York, pp. 85-125.

Ulrich R.S., Simons R., Losito B.D., Fiorito E., Miles M.A.,
Zelson M. 1991, Stress recovery during exposure to natural and urban environments, «Journal of Environmental Psychology», n.11, pp. 201-230.

van der Berg R. 2020, How Will COVID-19 Affect Urban Planning?, «TheCityFix», <https://thecityfix.com/blog/ will-covid-19-affect-urban-planning-rogier-van-denberg/>(08/20).

van der Berg A.E., Hartig T., Staats H. 2007, Preference for nature in urbanized societies: stress, restoration, and the pursuit of sustainability, «Journal of Social», n. 63, pp. 7996.

Weng M., Ding N., Li J., Jin X., Xiao H., He Z., Su, S. 2019, The 15-minute walkable neighbourhoods: measurement, social inequalities and implications for building healthy communities in urban China, "Journal of Transport \& Health», n. 13, pp. 259-273.

Wirth L. 1938, Urbanism as a way of life, «American Journal of Sociology», n. 44, pp. 1-24. 